\title{
FULLY NONLINEAR MODELING OF NEARSHORE WAVE PROPAGATION INCLUDING THE EFFECTS OF WAVE BREAKING
}

\author{
Christos E. Papoutsellis, Saint-Venant Hydraulics Lab., Cerema, Chatou, France, christos.papoutsellis@cerema.fr \\ Marissa L. Yates, Saint-Venant Hydraulics Lab., Cerema, marissa.yates-michelin@cerema.fr \\ Bruno Simon, Institut de Recherche sur les Phénomènes Hors Equilibre (IRPHE), Marseille, France, \\ simon@irphe.univ-mrs.fr \\ Michel Benoit, IRPHE \& Ecole Centrale Marseille, benoit@irphe.univ-mrs.fr
}

\section{INTRODUCTION}

Nearshore wave modeling over spatial scales of several kilometers requires balancing the fine-scale modeling of physical processes with the model's accuracy and efficiency. In this work, a fully nonlinear potential flow model is proposed as a compromise between simplified linear, weakly nonlinear or weakly dispersive models and direct CFD approaches.

The core of present approach is the use of a series representation for the velocity potential. This series contains prescribed vertical functions and allows the determination of the velocity potential in terms of unknown horizontal functions. The resulting dimensionally reduced model retains the structure of the Hamiltonian water wave system Zakharov (1968), Craig \& Sulem (1993), avoiding the solution of the Laplace problem for the potential. Instead, a numerically convenient linear system of horizontal equations needs to be solved at each step in the temporal evolution. No simplifications concerning the deformation of the physical boundaries are introduced, apart from the typical requirement of a smooth, non-overturning free surface and seabed. The main limitation of this formulation is its inability to account for wave breaking. The treatment of this process is the subject of the present work. Two different techniques are implemented in the present model. Simulation results are compared to laboratory measurements for two test cases: (1) shoaling and breaking of regular waves over a barred bathymetry Beji \& Battjes (1993) and (2) shoaling and breaking of regular waves on a plane beach Ting \& Kirby (1994).

\section{MODEL}

By considering an inviscid fluid and assuming that the flow is incompressible and irrotational, the wave motion is described in terms of the velocity potential and the free surface elevation. The kinematic and dynamic free surface boundary conditions are expressed as a system of evolution equations for the free surface elevation and the free surface velocity potential, which requires calculating the normal fluid velocity, or the Dirichlet-toNeumann (DtN) operator, at each time step Zakharov (1968), Craig \& Sulem (1993). The computation of the DtN operator is performed by using the Coupled-Mode method in the vertical direction Athanassoulis \& Papoutsellis (2017). The corresponding model, called Hamiltonian Coupled-Mode system (HCMS), has been successfully validated with comparisons to analytical solutions, laboratory experiments and other computational approaches for non-breaking waves, see e.g. Papoutsellis \& Athanassoulis (2017), Papoutsellis, Athanassoulis, \& Charalambopoulos (2017). Here, HCMS is extended to take into account the effects of depth-induced wave breaking.

When using potential flow theory, wave breaking cannot be modelled directly. The effects of wave breaking can however be included as a mechanism of wave energy dissipation that is activated by appropriate wave breaking criteria. Here, a criterion on the velocity of the free surface elevation, $\partial_{t} \eta$, is considered. A wave is assumed to start breaking when this velocity exceeds a specified value, proportional to the shallow water velocity: $\partial_{t} \eta>$ $\gamma \sqrt{g h}$, where $h$ is the local water depth and $\gamma$ is a constant depending on the configuration. A smaller threshold value is used for the termination of the breaking process Kennedy, Chen, Kirby, \& Dalrymple (2000). Once a breaking wave is detected, a dissipative term is triggered in the dynamic free surface boundary condition and is applied over a specified spatial region of the breaking wave. Two different methods are implemented and tested here: (1) an artificial pressure term proposed by Guignard \& Grilli (2001), acting in a region around the breaking crest and (2) an eddy viscosity term proposed by Kurnia \& van Groesen (2014), acting on the front face of the breaking wave.

\section{RESULTS}

The first test case evaluates the details of wave breaking over a barred bathymetric profile studied by Beji \& Battjes (1993) (BB93); see Figure 1, bottom. The incident wave height and period are $0.054 \mathrm{~m}$ and $2.5 \mathrm{~s}$ respectively, over a depth of $0.4 \mathrm{~m}$, and the breaking type is classified as plunging. The processes of shoaling, breaking and harmonic generation are reproduced well by both of the considered methods. Comparisons between the computed and measured free surface elevation are shown in Figure 1.

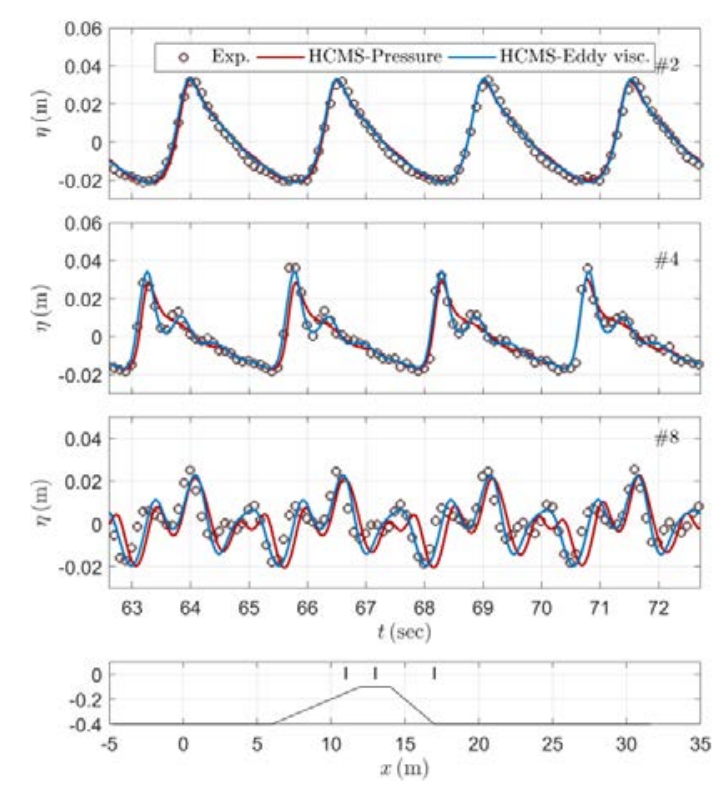

Figure 1. Comparison of simulated and free surface elevation at stations 2,4 , and 8 (top three panels), and bathymetric profile (bottom panel) for the plunging wave experiment of BB93. 
At station \#2, located in the shoaling region and close to the initial breaking point, the two wave breaking methods and the experimental results are nearly indistinguishable. Slight differences appear at stations \#4 and \#8, located in and after the breaking region, respectively.

The second test case investigates the shoaling and breaking of regular waves on a plane beach with slope $1 / 35$, following the experiments of Ting \& Kirby (1994) (TK94). For the spilling breaker case presented here, with incident wave height $0.0125 \mathrm{~m}$ and period $\mathrm{T}=2.0 \mathrm{~s}$ over an offshore depth of $0.4 \mathrm{~m}$, the models reproduce well the transition from symmetric to asymmetric waves with steeper wave faces and gentler rear slopes, and more peaked crests and wider troughs. The wave envelope (maximum and minimum free surface positions, Figure 2) and wave skewness and kurtosis (not shown here) are predicted well up to the breaking point. After the breaking point, the results obtained using the pressure-type dissipation term show slightly better agreement with the measurements of the maximum free surface elevation.

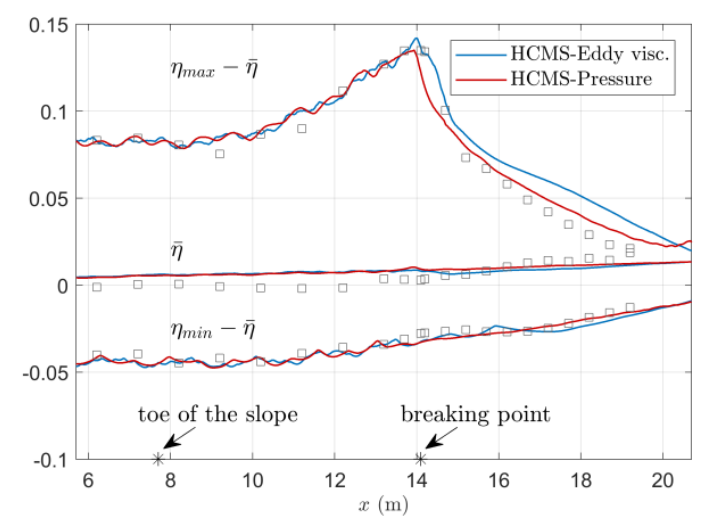

Figure 2. Comparison of simulated and measured wave envelopes along the wave channel for a spilling wave experiment of TK94.

\section{CONCLUSION}

The test cases presented here show the ability of the proposed model to simulate well the nonlinear processes of wave propagation and transformation over a barred beach and a mildly inclined beach. Breaking waves are detected by using a criterion on the velocity of the free surface elevation. In conjunction with this criterion, two wave-breaking techniques that successfully take into account the effects of breaking through wave energy dissipation, were evaluated and compared to experimental measurements of spilling and plunging breaking waves. Ongoing investigation and modification of the above approaches indicates that the simulation results can be improved by carefully selecting the spatial extent of the activation of the implemented wave breaking terms. Both methods can be extended to model domains with two horizontal dimensions. Investigation of the sensitivity of the above approaches on the choice of the involved free parameters will also be discussed and reported during the Conference and in the final paper.

\section{REFERENCES}

Athanassoulis. \& Papoutsellis (2017). Exact semiseparation of variables in waveguides with nonplanar boundaries. Proceedings of the Royal
Society A, 473:201700.

doi.org/10.1098/rspa.2017.0017

Beji \& Battjes (1993). Experimental investigation of wave propagation over a bar. Coastal Engineering, ELSEVIER, 19(1-2), 151-162. doi.org/10.1016/0378-3839(93)90022-Z

Craig \& Sulem (1993). Numerical Simulation of Gravity Waves. Journal of Computational Physics, ELSEVIER, 108, 73-83. doi.org/https://doi.org/10.1006/jcph.1993.1164

Guignard \& Grilli (2001). Modeling of wave shoaling in a 2D-NWT using a spilling breaker model. In ISOPE 2001. Stavanger, Norway.

Kenned, Chen, Kirby, \& Dalrymple (2000). Boussinesq modeling of wave tranformation breaking, and runup. I: 1d. Journal of Waterway, Port, Coastal, and Ocean Engineering, 126 39-47.

Kurnia \& van Groesen (2014). High order Hamiltonian water wave models with wave-breaking mechanism. Coastal Engineering, ELSEVIER, 93(1), 55-70. doi.org/10.1016/j.coastaleng.2014.08.002

Papoutsellis \& Athanassoulis (2017). A new efficient Hamiltonian approach to the nonlinear water-wave problem over arbitrary bathymetry. https://arxiv.org/abs/1704.03276

Papoutsellis, Athanassoulis \& Charalambopoulos (2017). Implementation of a fully nonlinear Hamiltonian Coupled-Mode Theory, and application to the interaction of solitary waves with varying bathymetry and vertical walls. Accepted in European Journal of Mechanics / B Fluids. arxiv.org/abs/1710.10847

Ting \& Kirby (1994). Observation of undertow and turbulence in a laboratory surf zone. Coastal Engineering,ELSEVIER, 24(1-2), 51-80. doi.org/10.1016/0378-3839(94)90026-4

Zakharov (1968). Stability of periodic waves of finite amplitude on the surface of a deep fluid. Zhurnal Prikladnoi Mekhaniki I Tekhnicheskoi Fiziki, 9(2), 86-94. 